\title{
Clinical outcomes of patients with multiple sclerosis treated with ocrelizumab in a US community MS center: an observational study
}

\author{
Kyle Smoot, ${ }^{1}$ Chiayi Chen (D) , ${ }^{2}$ Tamela Stuchiner, ${ }^{2}$ Lindsay Lucas, ${ }^{3}$ Lois Grote, ${ }^{2}$ \\ Stanley Cohan ${ }^{1}$
}

To cite: Smoot $\mathrm{K}$, Chen $\mathrm{C}$, Stuchiner T, et al. Clinical outcomes of patients with multiple sclerosis treated with ocrelizumab in a US community MS center: an observational study. BMJ Neurology Open 2021;3:e000108. doi:10.1136/ bmjno-2020-000108

Received 16 October 2020 Accepted 27 May 2021

Check for updates

(C) Author(s) (or their employer(s)) 2021. Re-use permitted under CC BY-NC. No commercial re-use. See rights and permissions. Published by BMJ.

${ }^{1}$ Providence Multiple Sclerosis Center, Providence Health and Services, Portland, Oregon, USA ${ }^{2}$ Providence Brain \& Spine Institute, Providence Health and Services, Portland, Oregon, USA ${ }^{3}$ NAMSA, Northwood, Ohio, USA

Correspondence to

Dr Kyle Smoot;

kyle.smoot@providence.org

\section{ABSTRACT}

Background To monitor long-term outcomes of ocrelizumab treatment.

Objective To evaluate safety and treatment outcomes of ocrelizumab in a community-based multiple sclerosis (MS) population.

Methods Adult patients with MS prescribed ocrelizumab were eligible. Chart reviews were conducted at the start of ocrelizumab treatment and every 6 months thereafter. Results Of the 355 patients enrolled, $71.9 \%$ were female; mean (SD) age was 51.8 (12.5) years; $78.3 \%$ had relapsing MS (RMS). Median baseline Expanded Disability Status Scale (EDSS) (IQR) was 3.0 (2.0-4.0) for RMS, 6.5 (6.0-7.5) for secondary progressive MS, and 6.5 (6.0-7.0) for primary progressive MS. Respiratory infections occurred in $40.1 \%$ and urinary tract infections in $33.1 \%$ of patients. There was no difference in the percentage of infections among patients $<55(68.5 \%, \mathrm{n}=122)$, and those $\geq 55$ of age $(67.5 \%, n=104)(p=0.94)$. Twenty-five hospitalisations were due to infections; $69.2 \%$ of these patients were $\geq 55$ with a mean EDSS of $5.7( \pm 1.86)$. Four patients have died. Serum IgM and IgG levels did not predict infection risk. Annualised relapse rate was 0.34 for the patients with RMS in the preceding 2 years and 0.09 in patients who received $\geq 2$ ocrelizumab $600 \mathrm{mg}$ courses. The first on-treatment MRI was stable in 262 (90.0\%) patients, $6.9 \%$ had new T2 lesions, $2.7 \%$ had enlarging T2 lesions and $1.4 \%$ had gadolinium-enhancing lesions. Median EDSS at 12 months was unchanged.

Conclusion 0crelizumab effectively controlled relapse risk and disability worsening. Although only $12.1 \%$ of patients have discontinued ocrelizumab, infections resulting in hospitalisation are a concern, especially in older and disabled patients.

\section{INTRODUCTION}

Ocrelizumab, a humanised anti-CD20 IgG antibody, induces complement-dependent and direct antibody-induced B cell lymphocyte destruction. ${ }^{1}$ Phase III trials showed ocrelizumab significantly reduced risk of relapse recurrence, 3 and 6 months sustained disability progression, gadolinium-enhancing $(\mathrm{Gd}+)$, and new/enlarging T2 lesions on MRI in patients with relapsing forms of multiple sclerosis (RMS) as compared with interferon $\beta$-la treated patients. ${ }^{2}$ In a placebo-controlled study of patients with primary progressive MS (PPMS), there was a statistically significant reduction in risk of 12-week sustained disability progression. ${ }^{3}$ Given these favourable results, ocrelizumab was approved in 2017 for treating RMS and PPMS, and has been prescribed for an increasing number of patients. ${ }^{4}$ While controlled trials are designed to generate class 1 evidence in regard to efficacy and risk of therapeutic agents, the criteria used for patient inclusion create potential selection bias. Because patients may be excluded due to an age limit, co-morbidities or concurrent medication use, data collected from these trials may not be generalisable to the broader disease population. In the phase III trials of ocrelizumab, patients older than 55 years of age were excluded, therefore, not addressing concerns about the risk of infection and other adverse events (AE) more commonly observed in older patients. In addition, given the relationship of hypogammaglobulinaemia and infections reported in patients treated with rituximab, we wanted to determine if this is a risk in our patients treated with ocrelizumab. ${ }^{5}$ The objective of the Providence Ocrelizumab Registry (POR) study is to determine whether use of ocrelizumab in a general MS population, which met U.S. Food and Drug Administration (FDA) prescribing indications for the treatment of MS, would reveal differences in clinical outcomes, especially their risk profile, when compared with that observed in controlled trials. 


\section{METHODS}

\section{Study design}

This was a prospective cohort study that used data collected from patients who participated in the POR. Patients were provided information about voluntary participation in the POR after their physicians prescribed ocrelizumab in accordance with the FDA approved label for the treatment of MS. Upon obtaining patient consent, demographic, clinical, MRI, medication history and laboratory data were collected through chart review by an experienced $\mathrm{RN}$ data abstractor at the time each patient started ocrelizumab and at each subsequent ocrelizumab $600 \mathrm{mg}$ treatment course.

\section{Setting and participants}

This ongoing registry enrolls patients at an outpatient MS centre located in Portland, Oregon, which consists of 5 clinicians and a team of clinical and research support staff treating over 2500 patients with MS. The Providence MS Center is part of a non-profit health system, providing care to an ethnically and socioeconomically diverse patient population throughout Oregon and Southwest Washington State.

Patients, aged 18 years and older, irrespective of sex, diagnosed with RMS, secondary progressive MS (SPMS) and PPMS, prescribed ocrelizumab between 28 March 2017 and 29 February 2020, and signed a research authorisation form to participate in the POR, were included in this study. There were no excluding criteria except for patients who did not sign the authorisation form, and they were not enrolled in the registry. Patients could withdraw their authorisation to participate in the registry at any time, but the data collected up to the time of withdrawal has remained in the database.

Demographic data collected include age, sex, race and ethnicity, date of symptom onset, date of diagnosis, history of heart disease, history of cancer, and MS pattern. Baseline data collected included the three most recently previously prescribed disease-modifying treatments (DMTs), relapses in the 24 months prior to starting ocrelizumab, MRI results prior to start of ocrelizumab, and Expanded Disability Status Scale (EDSS). Data collected after starting ocrelizumab included routine lab studies, AEs, hospitalisations, reason for discontinuation, relapses, MRI and EDSS.

Each chart review included all office visit notes, emergency room visits and hospital records. If a patient had healthcare encounters outside of the Providence Health System, those notes were made available for review either directly from the outside facility where care was provided or through the EPIC electronic medical record (EMR) Care Everywhere function, which, with patient consent, allows sharing of information in the EMR across clinics and hospitals. The occurrence of AEs of interest, including infections, hospitalizations and cancer, were collected at each clinic visit. Ocrelizumab $(600 \mathrm{mg})$ was administered intravenously every 6 months in a hospital or clinic infusion centre under supervision of medically qualified personnel. The first course included two infusions of ocrelizumab $300 \mathrm{mg}$ given 14 days apart.

\section{Clinical relapses, EDSS, laboratory assessment, and MRI results}

Each clinical relapse was verified at an in-person office visit by one of the treating neurologists of the Providence MS Center, each an expert in the diagnosis and treatment of MS. Clinical relapse was defined as new or worsening neurological symptoms, which persisted for more than 24 hours, in patients who had been clinically stable for 30 or more days before the event and was not attributable to other factors such as fever or infection. Although EDSS was not calculated at relapse assessment visits, each relapse required objective evidence of a change on neurological examination and/or MRI. The annualised relapse rate (ARR) was calculated by dividing the number of relapses by the number of years on medication for each patient.

Baseline EDSS scores were obtained, by the treating physician, prior to the first course of ocrelizumab, and at yearly clinic visits. EDSS was not calculated for other clinic visits. All the examiners were level C Neurostatuscertified Kappos 2009 revision EDSS raters.

Laboratory testing, including hepatic panel, complete cell count and immunoglobulin levels, was performed prior to starting ocrelizumab and within a month prior to each subsequent infusion.

The MRIs were performed prior to initiating ocrelizumab, 6 months after starting ocrelizumab and yearly thereafter. Imaging was performed on a 1.5 or $3.0 \mathrm{~T}$ MRI scanner, using a standardised MS protocol, with $5 \mathrm{~mm}$ contiguous gap-free slices of the brain. Neuroradiologists with extensive experience in diagnostic imaging for MS interpreted the scans. Brain MRI sequences included T1-weighted and T2-weighted axial, fluid-attenuated inversion recovery axial and sagittal, diffusion-weighted axial and gadolinium-enhanced T1-axial views and axial diffusion weighted images. MRI changes were defined as new or enlarging T2 lesions and/or presence of gadoliniumenhancing $(\mathrm{Gd}+) \mathrm{T} 1$ lesions. For spinal MRIs, sagittal T2 imaging was performed with a $3 \mathrm{~mm}$ slice thickness and a $4 \mathrm{~mm}$ gap, and axial T2 imaging consisted of volumetric acquisition, reconstructed at $1 \mathrm{~mm}$ interval with $0.5 \mathrm{mg}$ gap.

\section{Statistical analysis}

Demographics, prior DMT use, ARR for 24 months prior to starting ocrelizumab, number of ocrelizumab courses received, EDSS, AEs, hospitalisations, time on ocrelizumab, serum IgM/IgG levels and ocrelizumab discontinuations were summarised as percentages, means (SD) or medians $(\mathrm{IQR}=(\mathrm{Q} 1, \mathrm{Q} 3))$ as appropriate. Bivariate analyses were performed using chi-square and t-test to determine if there were any differences between patients who relapsed and those who did not, as well as for those who did and did not have infections. Paired t-test were used to determine if there were improvements over time 
in Modified Fatigue Impact Scale (MFIS) and the Beck Depression Inventory-II (BDI-II) comparing baseline and 12 months. SPSS V.26 (IBM Corp.) was used for analysis.

To examine the effect of serum immunoglobulin (Ig) levels on the risk of infection, analyses were conducted separately for OCR courses starting at 6, 12 and 18 months. For each course, the Ig levels collected immediately before OCR treatment were included in logistic regression analyses as potential predictors for the risk of infection developed during the 6-month course following OCR treatment. Low IgG levels were defined as IgG $\leq 680 \mathrm{mg} / \mathrm{dL}$ and low IgM levels as IgM $\leq 45 \mathrm{mg} / \mathrm{dL}$. A total of six univariate and multivariable logistic regression analyses were conducted: one per course for both $\operatorname{IgG}$ and $\operatorname{IgM}$ as the predictors of interest. Proportions of subjects who developed infections as well as unadjusted and adjusted ORs with 95\% CIs and associated $\mathrm{p}$ values are presented. Multivariable logistic regression analyses included age ( $\leq 55 \mathrm{vs}>55$ ), sex, previous DMT (treatment naïve vs at least one DMT), disease duration, and MS pattern (PMS vs not PMS) as the covariates. For each analysis, only subjects with complete outcome and covariate data were included.

Patients missing lab values, EDSS, imaging, MFIS or BDI-II scores were excluded from those analyses.

\section{RESULTS}

\section{Demographics}

Of 355 patients enrolled, $71.9 \%$ were female, $90.5 \%$ were white, $78.3 \%$ had RMS, $13.2 \%$ had SPMS, and $8.5 \%$ had PPMS. The mean (SD) age was 51.8 (12.5) years (table 1$).$ The median duration of disease was 12 years (IQR: 6.5-18.4) for RMS, 18.7 years (IQR: 12.1-23.7) for SPMS, and 12.7 years (IQR: 7.7-17.2) for PPMS (table 2).

Almost $50 \%$ of patients switched from natalizumab (NTZ) (18.9\%), dimethyl fumarate $(15.1 \%)$ or fingolimod (15.1\%), and $27.4 \%$ were treatment naïve. Median time on ocrelizumab (IQR) was 23.6 (19.0-29.7) months. Approximately $59 \%$ of patients had been on ocrelizumab for 2 years or more. Of 332 patients who had two or more $600 \mathrm{mg}$ courses of ocrelizumab, more than $85 \%$ have had at least four chart reviews since their first infusion.

\section{Safety}

Infusion reactions were more prevalent in patients receiving the initial course of ocrelizumab and became less frequent with each subsequent infusion (figure 1). Other common AEs were respiratory infections, occurring in $40.1 \%$, and urinary tract infections (UTI) in $33.1 \%$ of patients (table 3 ). There was no difference in the percentage of infections among patients under the age of $55(68.5 \%, \mathrm{n}=122)$, and those 55 or older $(67.5 \%$, $\mathrm{n}=104) \quad(\mathrm{p}=0.94)$. Sixty-three hospitalisations occurred in 37 patients; 24 of the hospitalizations resulted from infection, the majority being UTIs (63\%) and five of these patients developed urosepsis. Sixty-nine percent of hospitalised patients were 55 years or older (mean age
Table 1 Patientcharacteristics

\begin{tabular}{|c|c|}
\hline Number of patients & 355 \\
\hline Mean age in years (SD) & $51.8( \pm 12.5)$ \\
\hline Female, n (\%) & $256(71.9)$ \\
\hline Median disease duration in years (IQR) & $13.1(7.6,19.3)$ \\
\hline \multicolumn{2}{|l|}{ Race/ethnicity, n (\%) } \\
\hline White & $315(90.5)$ \\
\hline Non-white & $33(9.5)$ \\
\hline \multicolumn{2}{|l|}{ MS pattern, n (\%) } \\
\hline RMS & $278(78.3)$ \\
\hline SPMS & 47 (13.2) \\
\hline PPMS & $30(8.5)$ \\
\hline \multicolumn{2}{|l|}{ Previous DMT, n (\%) } \\
\hline Interferon & $11(3.1)$ \\
\hline Glatiramer acetate & $23(6.6)$ \\
\hline Fingolimod & $53(15.1)$ \\
\hline Dimethyl fumarate & $53(15.1)$ \\
\hline Teriflunomide & $20(5.7)$ \\
\hline Natalizumab & 66 (18.9) \\
\hline Other $\mathrm{Med}^{\star}$ & $28(8.0)$ \\
\hline Treatment naive & $96(27.4)$ \\
\hline Median time in months on OCR (IQR) & 23.6(19.0, 29.7) \\
\hline Time on drug 1 year or less, n (\%) & 45 (12.7) \\
\hline Time on drug 1 to 2 years, $n(\%)$ & $101(28.5)$ \\
\hline Time on drug 2 years or more, n (\%) & $209(58.9)$ \\
\hline $\begin{array}{l}\text { Patients with Infusion reactions-dose } 1 \text {, } \\
\mathrm{n}(\%)\end{array}$ & 125 (35.2) \\
\hline Reaction requiring treatment, $\mathrm{n}(\%)$ & $89(25.1)$ \\
\hline $\begin{array}{l}\text { Patients with infusion reactions- } \\
\text { subsequent doses, } † \mathrm{n}(\%)\end{array}$ & $67(18.9)$ \\
\hline Reaction requiring treatment, $\mathrm{n}(\%)$ & $43(12.1)$ \\
\hline Lost to follow-up, n (\%) & $23(6.5)$ \\
\hline Discontinued, n (\%) & $43(12.1)$ \\
\hline
\end{tabular}

*Other med includes: mycophenolate, intravenous methylprednisolone, daclizumab, cyclophosphamide, azathioprine, and study drugs through clinical trials.

†Among patients who had more than one dose or a 6 month review following first dose $(n=337)$.

DMT, disease-modifying treatment; OCR, ocrelizumab; PPMS, primary progressive multiple sclerosis; RMS, relapsing multiple sclerosis; SPMS, secondary progressive multiple sclerosis.

$58.2 \pm 12.5)$. The mean EDSS of those who were hospitalised was $5.7( \pm 1.86)$. Thirty-two patients $(9 \%)$ had a history of cancer, and eight patients were newly diagnosed with cancer after starting ocrelizumab (basal cell (4), breast cancer (2), each diagnosed at 7 and 23 months after starting ocrelizumab), recurrent thyroid cancer (1), and melanoma (1)). Forty-three (12\%) patients discontinued ocrelizumab after a median duration (IQR) of 10.8 (6.1-18.9) months, 24 due to side effects, 15 due 
Table 2 Patient characteristics by multiple sclerosis (MS) pattern

\begin{tabular}{llcc}
\hline & RMS & SPMS & PPMS \\
& N (\%) & N (\%) \\
\hline & $278(78.3)$ & $47(13.2)$ & $30(8.5)$ \\
\hline Median disease duration in years (IQR) & $12.0(6.5-18.4)$ & $18.7(12.1-23.7)$ & $12.7(7.7-17.2)$ \\
\hline Mean number of doses (SD) & $3.9(1.7)$ & $4.1(1.6)$ & $3.9(1.5)$ \\
\hline Median time in months on OCR (IQR) & $24.8(16.8-29.5)$ & $28.7(23.4-30.4)$ & $26.0(23.4-29.8)$ \\
\hline ARR 24 months prior to OCR (SD) & $0.34( \pm 0.42)$ & $0.14( \pm 0.34)$ & 00 \\
\hline Previous DMT, \% (n) & & & 0 \\
\hline Interferon & $9(3.3)$ & $2(4.3)$ & $1(3.4)$ \\
\hline Glatiramer acetate & $18(6.6)$ & $4(8.5)$ & $2(6.9)$ \\
\hline Fingolimod & $45(16.4)$ & $6(12.8)$ & $2(6.9)$ \\
\hline Dimethyl fumarate & $47(17.2)$ & $4(8.5)$ & $2(6.9)$ \\
\hline Teriflunomide & $18(6.6)$ & $2(4.3)$ & $2(6.9)$ \\
\hline Natalizumab & $59(21.5)$ & $5(10.6)$ & $5(17.2)$ \\
\hline Other Med* & $19(6.9)$ & $4(8.5)$ & $17(58.6)$ \\
\hline Treatment naive & $59(21.5)$ & $20(42.6)$ & $6.5(6.0-7.0)$ \\
\hline Median baseline EDSS (IQR) & $3.0(2-4)$ & $6.5(6-7.5)$ & $6.5(5.6-7.5)$ \\
\hline Median EDSS (IQR) at 12 months (n) & $3.0(2.0-5.5)(151)$ & $6.5(6.5-7.5)(31)$ & $(16)$ \\
\hline
\end{tabular}

${ }^{*}$ Other Med includes mycophenolate, intravenous methylprednisolone, daclizumab, cyclophosphamide, azathioprine, and study drugs through clinical trials.

EDSS, Expanded Disability Status Scale; PPMS, primary progressive MS; RMS, relapsing MS; SPMS, secondary progressive MS.

to a relapse or clinical progression, and 4 due to death (table 4).

The first death occurred in a 58-year-old patient with RMS and a baseline EDSS of 6.04 months after the second course of ocrelizumab at 6 months. The patient reported increased shortness of breath, declined to be seen at the

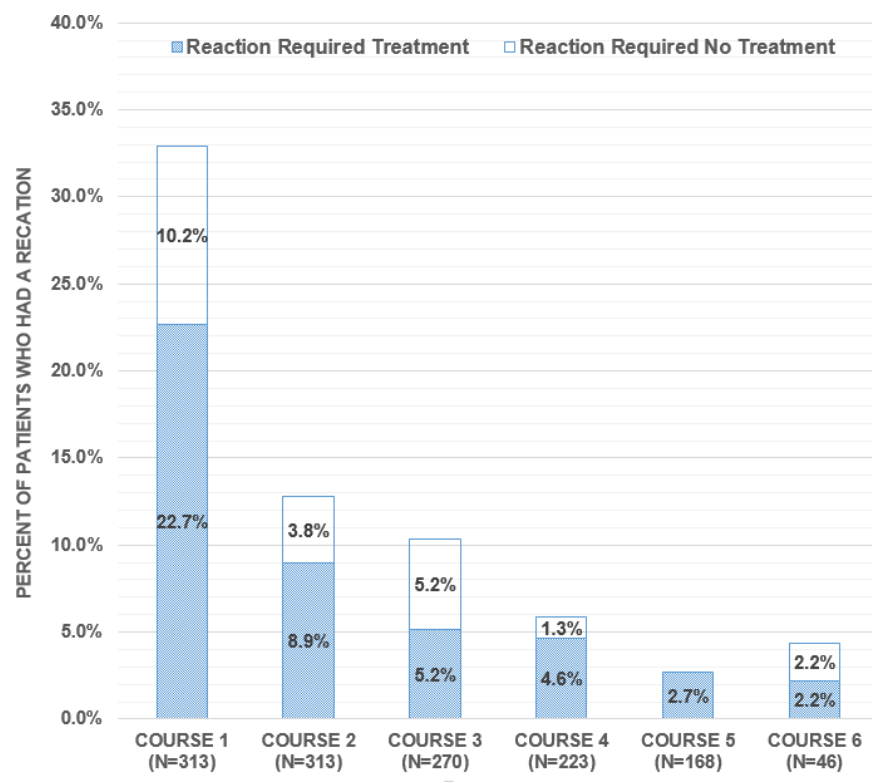

Figure 1 Infusion reactions by ocrelizumab course*. *represents only those patients with at least two courses (course 1 includes first and second $300 \mathrm{mg}$ infusions). emergency department, and was found dead at home the following day. The second death was a 56-year-old with SPMS and a baseline EDSS of 7.5. The patient died just prior to receiving the scheduled 12-month infusion of ocrelizumab due to urosepsis. The patient had multiple hospitalisations for treatment of infections during the 2 months prior to their death. The third death was a 45-year-old patient with RMS and a history of severe depression, who committed suicide 3 months after their 2 baseline $300 \mathrm{mg}$ ocrelizumab infusions. The fourth death

\begin{tabular}{ll}
\hline Table $3 \quad$ Infections & \\
\hline $\mathrm{N}^{*}$ & 355 \\
$\begin{array}{l}\text { Age of patients who had infection, } \\
\text { median (IQR) }\end{array}$ & $53.5(42.5-62.7)$ \\
\hline Patients who had infection(s), $\mathrm{n}(\%)$ & $228(63.9)$ \\
\hline Upper respiratory infection & $143(40.1)$ \\
\hline Urinary tract infection & $119(33.1)$ \\
\hline Bronchitis & $3(1.0)$ \\
\hline Pneumonia & $11(3.1)$ \\
\hline Shingles & $7(2.0)$ \\
\hline Genital herpes & $5(1.4)$ \\
\hline Sepsis & $8(2.2)$ \\
\hline Other infection & $50(14.0)$ \\
\hline
\end{tabular}

*All patients who had at least one dose of OCR. 
Table 4 Discontinuation of ocrelizumab

\begin{tabular}{|c|c|}
\hline Patients who discontinued, n (\%) & $43(12.1)$ \\
\hline $\begin{array}{l}\text { Median time in months to discontinuation } \\
\text { (IQR) }\end{array}$ & $10.8(6.1-18.9)$ \\
\hline Median age of those discontinued (IQR) & $57.8(45.8-63.7)$ \\
\hline \multicolumn{2}{|l|}{ Reasons for discontinuation*, n (\%) } \\
\hline Pregnancy & $2(4.7)$ \\
\hline Cost/insurance issues & $2(4.7)$ \\
\hline Side effects & $24(55.8)$ \\
\hline Safety concerns/issues & $13(30.2)$ \\
\hline Relapse & $6(14.0)$ \\
\hline Clinical progression & $9(20.9)$ \\
\hline Death & $4(9.3)$ \\
\hline $\begin{array}{l}\text { Among those who discontinued due to } \\
\text { side effects } \dagger, \mathrm{n}(\%)\end{array}$ & $\mathrm{N}=24$ \\
\hline Recurrent infections & $9(39.1)$ \\
\hline Fatigue/malaise & $13(56.5)$ \\
\hline Weakness & $3(13.0)$ \\
\hline Anxiety/depression & $3(13.0)$ \\
\hline Headache & $2(8.7)$ \\
\hline Sore throat & $2(8.7)$ \\
\hline Respiratory issues & $3(13.0)$ \\
\hline Cancer & $2(8.7)$ \\
\hline Hair loss & $1(4.3)$ \\
\hline
\end{tabular}

*Except for death, some patients had more than one reason given for discontinuation.

†Some patients discontinued due to more than one side effect.

was a 61-year-old patient with RMS who died of pneumonia and septic shock 2 months after receiving the 2 baseline $300 \mathrm{mg}$ ocrelizumab infusions.

Grade III lymphopenia occurred in three patients with a mean age (SD) of $67.2(0.459)$ years compared with 52.6 (12.4) in patients without grade III lymphopenia. Seventy-seven patients $(27.0 \%)$ had at least one absolute lymphocyte count of 0.50 to 0.99 that indicated grade I or II lymphopenia while on ocrelizumab.

Three hundred and thirty-seven patients $(88.7 \%)$, who had at least one $\operatorname{IgM} / \operatorname{IgG}$ value measured and received at least two courses of ocrelizumab, were included in the immunoglobulin analyses. Serum $\mathrm{IgG} / \mathrm{IgM}$ levels were obtained within a month before each ocrelizumab infusion. The median time on ocrelizumab (IQR) was 26 months (21-29.8). Infections were seen in 226 of the 337 patients $(67.1 \%)$. The median age (IQR) for those who had an infection was 53.5 (42.5-62.7) and was 53.0 (42.260.2) for those who did not.

Logistic regression analyses showed no evidence of a higher risk of infection for subjects with low IgG or IgM levels. After adjusting for covariates, the likelihood of infection during the 6-month course following OCR treatment was not significantly different for subjects with high vs low IgG levels at course 2 (adjusted odds ratio $(\mathrm{AOR})=1.41$ (95\% CI 0.64-3.23); $\mathrm{p}=0.399)$, course $3(\mathrm{AOR}=0.53(95 \%$ CI $0.24,1.15) ; \mathrm{p}=0.110)$, and course $4(\mathrm{AOR}=0.62(95 \%$ CI $0.26,1.51) ; \mathrm{p}=0.295) \quad($ table 5$)$. It was also not significantly different for subjects with high vs low IgM levels at course $2(\mathrm{AOR}=0.84$ (95\% CI 0.39, $1.81) ; \mathrm{p}=0.644)$, course 3 (AOR $=0.61(95 \%$ CI $0.28,1.29)$; $\mathrm{p}=0.201)$, and course $4(\mathrm{AOR}=1.51(95 \%$ CI $0.65-3.60)$; $\mathrm{p}=0.341) \quad($ table 6).

\section{Efficacy}

On-ocrelizumab ARR was 0.09 as compared with 0.34 over the 24 months prior to starting ocrelizumab for the RMS cohort. Thirty-three of the 332 patients who had at least two courses of ocrelizumab relapsed. Almost $28 \%$ of the relapses occurred in patients transitioning from NTZ; however, the rate of relapse was not significantly higher than that of the other DMT groups. The patients who transitioned from NTZ had a mean (SD) washout period of $4.4( \pm 1.4)$ weeks and a median time (IQR) to first relapse of 8.7 (3.3-13.9) months. Bivariate tests for age, disease duration, sex, pre-drug ARR, number of previous DMTs used and prior DMT used demonstrated

Table 5 ORs and 95\% confidence intervals for infection by lgG level

\begin{tabular}{|c|c|c|c|c|c|}
\hline & & $\mathbf{N}$ & Infection (\%) & Unadjusted OR $(95 \% \mathrm{Cl})^{*}$ & Adjusted OR (95\% Cl)* \\
\hline \multicolumn{6}{|c|}{ Dependent: Infection between 6 and 12 months of OCR treatment $(n=165)$} \\
\hline 6 -month lgG & $\leq 680$ & 38 & 31.6 & Reference & Reference \\
\hline \multicolumn{6}{|c|}{ Dependent: infection between 12 and 18 months of OCR treatment $(n=150)$} \\
\hline 12-month IgG & $\leq 680$ & 42 & 50.0 & Reference & Reference \\
\hline \multicolumn{6}{|c|}{ Dependent: infection between 18 and 24 months of OCR treatment $(n=122)$} \\
\hline \multirow[t]{2}{*}{ 18-month lgG } & $\leq 680$ & 38 & 42.1 & Reference & Reference \\
\hline & $>680$ & 84 & 32.1 & 0.65 (0.30 to 1.45$) p=0.288$ & 0.62 (0.26 to 1.51$) p=0.295$ \\
\hline
\end{tabular}

${ }^{*}$ Based on univariate and multivariable logistic regression analyses for infection. Multivariable logistic regression analyses included covariates age ( $\leq 55$ vs $>55$ ), previous DMT (treatment naïve vs at least one DMT), disease duration, MS pattern (PMS vs not PMS) and sex. 
Table 6 ORs and 95\% Cls for Infection by lgM level

N Infection (\%) Unadjusted OR $(95 \% \mathrm{Cl})^{*} \quad$ Adjusted OR $(95 \% \mathrm{Cl})^{*}$

\begin{tabular}{|c|c|c|c|c|c|}
\hline \multicolumn{6}{|c|}{ Dependent: infection between 6 and 12 months of OCR treatment $(n=159)$} \\
\hline \multirow[t]{2}{*}{ 6-month IgM } & $\leq 45$ & 47 & 36.2 & Reference & Reference \\
\hline & $>45$ & 112 & 37.5 & 1.06 (0.53 to 2.18$) p=0.874$ & $0.84(0.39$ to 1.81$) p=0.644$ \\
\hline \multirow[t]{2}{*}{ 12-month IgM } & $\leq 45$ & 51 & 43.1 & Reference & Reference \\
\hline & $>45$ & 95 & 37.9 & $0.80(0.40$ to 1.61$) p=0.537$ & $0.61(0.28$ to 1.29$) p=0.201$ \\
\hline \multirow[t]{2}{*}{ 18-month IgM } & $\leq 45$ & 55 & 30.9 & Reference & Reference \\
\hline & $>45$ & 64 & 39.1 & $1.43(0.67$ to 3.10$) p=0.354$ & $1.51(0.65$ to 3.60$) p=0.341$ \\
\hline
\end{tabular}

*Based on univariate and multivariable logistic regression analyses for infection. Multivariable logistic regression analyses included covariates age ( $\leq 55$ vs $>55$ ), previous DMT (treatment naïve vs at least one DMT), disease duration, MS pattern (progressive vs non progressive MS) and sex.

no significant differences between those who did or did not relapse (data not shown).

Brain MRIs prior to ocrelizumab initiation were obtained in 347 of 355 patients, with median time of 3.0 months (IQR: 1.2-5.9) between the last preocrelizumab MRI and first ocrelizumab dose. First follow-up MRIs were obtained in $291(81.9 \%)$ patients 5.6 (4.4-7.7) months after the first ocrelizumab course, $76 \%$ solely brain MRI, $19 \%$ brain plus spinal cord, and $5 \%$ spinal cord only. Of the 291 patients with post-ocrelizumab scans, $262(90.0 \%)$ had stable MRI results. Enhancing lesions were seen in 4 (1.4\%) patients. Insurer refusal was primarily responsible for those patients who did not obtain MRI scans within the first year after starting ocrelizumab.

Baseline median EDSS (IQR) for RMS, SPMS and PPMS subgroups was 3.0 (2.0-4.0), 6.5 (6-7.5), and 6.5 (6.0-7.0), respectively, and remained unchanged at 12 months (table 2). In addition to the favourable ARR and MRI outcomes, treatment efficacy was also evidenced by the absence of disability progression as measured by EDSS.

There were no significant differences in BDI from baseline to 12 months $(n=88$, mean difference $-0.61 \quad( \pm 7.6)$, $\mathrm{p}=0.4)$, but MFIS score improved at 12 months $(\mathrm{n}=87)$, with a mean difference of $-3.7( \pm 14.1)(\mathrm{p}=0.02)$.

\section{DISCUSSION}

Given the age and comorbidity restrictions in the phase III clinical trials, our registry was established to evaluate safety and clinical outcomes in a community-based population of patients treated with ocrelizumab, with the hope of studying a cohort more consistent with a realworld population of eligible patients with MS. In terms of safety, infusion reaction rates in the POR cohort did not significantly differ from those observed in the prior phase III clinical trials, the first course having the highest rate followed by a stepwise decline with subsequent ocrelizumab infusions. In our cohort, $32.9 \%$ of patients had a reaction with course 1 compared with $32.2 \%$ in the phase
III clinical trials. The most common infections were URIs, also consistent with the findings from the phase III controlled clinical trials. Although patients older than 55 were not at higher risk of having an infection, the number of hospitalisations in older patients in our cohort due to an infection is concerning, the majority of those being secondary to UTIs. The mean EDSS was $5.7( \pm 1.86)$, and $69 \%$ of these patients were older than 55. Given these latter observations, we recommend that the patient's age and level of disability should be taken into account when considering the use of ocrelizumab and should prompt very close monitoring when it is employed in older and more disabled patients. Although there were 12 cases of herpes infections detected, there were no cases of systemic or CNS involvement. No cases of progressive multifocal leukoencephalopathy or other significant safety issues have been observed.

In June 2019, the European Medicines Agency updated the ocrelizumab prescribing information to include the association between a reduction in serum immunoglobulin levels, especially IgG, and serious infections. More recently, similar information was added to the prescribing information in the USA. ${ }^{4}$ However, our data, at this time, do not demonstrate a correlation between serum IgM/ IgG levels and risk of infection. The median time on ocrelizumab was a little less than 2 years, so a longer observation period may eventually corroborate an association between immunoglobulin levels and the risk of serious infection.

In terms of efficacy, the data from our cohort revealed that ocrelizumab is effective in controlling relapses with an ARR of 0.09. Although our RMS data are consistent with the ARR of 0.16 reported in OPERA1 and OPERA $2,{ }^{2}$ caution is advised when comparing results from controlled studies that were conducted in different patient populations and with different study designs. To date, $43(12.1 \%)$ patients have discontinued ocrelizumab, $15(4.2 \%)$ solely due to a relapse or clinical progression. Of the relapses, $63.6 \%$ occurred after 6 or more months 
on ocrelizumab therapy. There were no predicting factors for a relapse, but patients transitioning from NTZ had the highest occurrence of relapse $(27.3 \%)$, while comprising only $18.6 \%$ of the total ocrelizumab-treated cohort. This occurred despite a mean interval of only $4.4( \pm 1.4)$ weeks from their last NTZ dose. This observation was surprising given that previously published data have indicated an increased risk of relapses 8-12 or more weeks after the last dose of NTZ. ${ }^{67}$ Also, the median time to first relapse after transitioning from NTZ was 8.7 months; therefore, OCR should have been fully effective by that time.

To date, there has been no evidence of disability progression in our ocrelizumab-treated patients with RMS, SPMS, or PPMS as median EDSS scores have remained stable up to 30 months since starting ocrelizumab. Follow-up MRIs were also stable in $90.2 \%$ of patients.

Registries and open-label extension studies play an important role in acquiring long-term safety, and tolerability data from a broader patient population as compared with the more homogeneous patient cohorts typically studied in controlled clinical trials. Although post-approval AE reporting is an important source of information relating to risk, unlike registries and longterm open label studies, this information is often incomplete, likely under-reporting AEs. Limitations of registries include having multiple clinicians, which potentially introduces variability in the timing, frequency and results of clinical assessments. However inter-rater variability in this study was minimised because of prespecified relapse and disability progression criteria, the level of expertise of the examiners, and MRI consensus between neuroradiologists and neurologists. Another shortcoming is that timing and frequency of MRI are often less than ideal in community-based cohorts, mainly because of delay or refusal of insurance authorisations, or lack of patient financial resources. Although the MRI scans collected in our registry followed a strict protocol, we could not prespecify that patient be scanned on the same machine each time, and not all patients had their MRI scans done at a Providence facility, due to geographical or insurance constraints. Absence of a blinded examiner may also have introduced bias on clinical outcome assessment.

Despite these potential shortcomings, we believe that this study provides valuable information concerning the use of ocrelizumab in a large community-based MS centre, and may assist in future decision-making regarding the selection and management of patients who are potential candidates for ocrelizumab therapy. Although only 355 patients had been enrolled in the POR as of February
2020, the number of patients in this registry has continued to grow, which will allow for our centre to monitor tolerance, and risks of ocrelizumab administration over time and gain a broader perspective of this important therapeutic agent.

Contributors KS, CC, TS and SC contributed to the planning and the conduct of the study. LG contributed to the collection and the reporting of the data. TS and LL contributed to the statistical analysis. KS, CC, TS and SC also contributed to the writing of the manuscript.

Funding The authors have not declared a specific grant for this research from any funding agency in the public, commercial or not-for-profit sectors.

Competing interests KS has received institutional research support from AbbVie, Biogen, Genentech, EMD Serono, MedDay, and IMS Health and consulting fees from Acorda, Biogen, EMD Serono, Genzyme, Genentech, Novartis, and Teva. SC has served on advisory boards or steering committees for AbbVie, Biogen, Bristol Myers Squibb (Celgene), Novartis, Sanofi Genzyme, and Pear Therapeutics; has received institutional research support from AbbVie, Adamas, Biogen, EMD Serono, Novartis, Sanofi Genzyme, MedDay, and Roche Genentech; has received speaker honoraria from AbbVie, Biogen, Novartis, Sanofi Genzyme, and Roche Genentech.

Patient consent for publication Not required.

Ethics approval Providence Health \& Services IRB STUDY2016000086. This study is supported solely by the Providence Brain and Spine Institute and was approved by the Providence Institutional Review Board.

Provenance and peer review Not commissioned; externally peer reviewed. Data availability statement Data are available upon reasonable request.

Open access This is an open access article distributed in accordance with the Creative Commons Attribution Non Commercial (CC BY-NC 4.0) license, which permits others to distribute, remix, adapt, build upon this work non-commercially, and license their derivative works on different terms, provided the original work is properly cited, appropriate credit is given, any changes made indicated, and the use is non-commercial. See: http://creativecommons.org/licenses/by-nc/4.0/.

\section{ORCID iD}

Chiayi Chen http://orcid.org/0000-0001-6636-5203

\section{REFERENCES}

1 Martin F, Chan AC. B cell immunobiology in disease: evolving concepts from the clinic. Annu Rev Immunol 2006;24:467-96.

2 Hauser SL, Bar-Or A, Comi G, et al. Ocrelizumab versus interferon beta-1a in relapsing multiple sclerosis. $N$ Engl J Med 2017;376:221-34.

3 Montalban X, Hauser SL, Kappos L, et al. Ocrelizumab versus placebo in primary progressive multiple sclerosis. N Engl J Med 2017;376:209-20.

4 Genentech Inc. Ocrevus: highlights of prescribing information, 2018.

5 Barmettler S, Ong M-S, Farmer JR, et al. Association of immunoglobulin levels, infectious risk, and mortality with rituximab and hypogammaglobulinemia. JAMA Netw Open 2018;1:e184169.

6 Sellner J, Rommer PS. A review of the evidence for a natalizumab exit strategy for patients with multiple sclerosis. Autoimmun Rev 2019;18:255-61.

7 Cohan SL, Moses H, Calkwood J, et al. Clinical outcomes in patients with relapsing-remitting multiple sclerosis who switch from natalizumab to delayed-release dimethyl fumarate: a multicenter retrospective observational study (strategy). Mult Scler Relat Disord 2018;22:27-34. 